\title{
RESPONSIVE BISIMULATION
}

\author{
Xiaogang Zhang and John Potter \\ School of Computer Science and Engineering \\ University of New South Wales, Australia \\ \{xzhang,potter\}@cse.unsw.edu.au
}

\begin{abstract}
This paper introduces the responsive bisimulation, which treats local delays of incoming messages the same as external delays, as long as potential interference by competing receptors is avoided. By this bisimulation, the $\pi$-calculus process $k . m . P$, representing a lock $k$ with a message receptor $m$, is equivalent to the process $(\nu n)(m . \bar{n} \mid k . n . P)$; the first process will delay messages externally, the second locally. Existing bisimulations distinguish between these processes. The responsive bisimulation is a congruence for the family of processes which model objects. It is useful for studying compositional synchronisation in such models.
\end{abstract}

\section{Introduction}

With the ability to directly model dynamic reference structures, process algebras such as the $\pi$-calculus ([Milner92, Milner99]) and its variations have been used to model concurrent objects ([Walker95, Jones93, Sangi96, Hüttel96, Zhang97]). Some researchers ([Schne97, Zhang98a, Zhang98b]) have also applied it to model compositional concurrent objects in the aspect-oriented programming style ([Aksit92, Holmes97]) attempting to avoid the inheritance anomaly [McHale94]. One of the important issues with such models is to identify the similarity between composed behaviours and the expected behaviour. There are many known bisimulation techniques available for various purposes. For example, the weak ground bisimulation and many others recognise equivalence of processes $(\nu n)(m . \bar{n} \mid n . P)$ and $m . P$, by ignoring the internal forwarding. This paper presumes familiarity with the $\pi$-calculus and its bisimulation relations.

Existing bisimulations distinguish between processes $(\nu n)(m . \bar{n} \mid k . n . P)$ and $k . m . P$, the former represents an object with a lock $k$ and a forwarder (via $n$ ) for message $m$ so that any delays in servicing a method call are local, whereas the latter process causes an external delay in reception of $m$. We want to treat these two processes as equivalent, since their behaviour in emitting responses to incoming messages is the same.

In this paper we propose the notion of responsive bisimulation to capture this kind equivalence by allowing the location of input delays to be ignored. This is different from asynchronous bisimulation which is insensitive to output delays and considers $(m \cdot \bar{m} \mid P)$ to be the same as $P$; we detail the differences in Section 6 . We can take 
alternative views of responsive bisimulation. First, from the target process, we can tolerate delays of incoming messages at input ports when determining the similarity between process evolution trees. Second, when testing a process, we can "localise" messages by buffering them in the environment, and make them only accessible to the target process. External observers cannot see these localised messages, so cannot mistake them as output from the target process. In Section 4 we will show these views are equivalent.

One of the major results of this paper is that the responsive bisimulation is a congruence for the family of processes which model objects, that is, the processes which uniquely own all the message receptors they use. All process combinators, except replication and parallel composition, always preserve responsive bisimulation. Replication preserves responsive bisimilarity for the family of processes which model objects, and parallel composition preserves it for an even larger family of processes.

Another interesting result, revealed in Proposition 20, is that, a persistently available receptor with an internal forwarder can be ignored.

The rest of the paper is structured as follows: Section 2 motivates this work; Section 3 briefly introduces the polar $\pi$-calculus and related notions; Section 4 defines responsive bisimulation; Section 5 gives some properties of the equivalence and other theoretical results; Section 6 discusses some further issues relating the responsive bisimulation with other notions; and Section 7 concludes the paper. Most proofs are omitted here in order to save space; interested readers can find them in [Zhang02b].

\section{Background}

In [Zhang98a] and [Zhang98b], the behaviour of a concurrent object is modelled using the $\pi$-calculus as the parallel composition of a process $F$, representing the object's functional behaviour with no constraint on its concurrent interactions, and a process $C$ representing the constraints on the object's concurrent behaviour. For example, the functionality of a buffer object can be described by $F \stackrel{\text { def }}{=} ! n_{r}(x) \cdot M_{r}\langle x\rangle \mid ! n_{w}(x) . M_{w}\langle x\rangle$, where $n_{r}(x) . M_{r}\langle x\rangle$ and $n_{w}(x) . M_{w}\langle x\rangle$ represent the behaviour of the read and write methods respectively; each of them can have unlimited invocations executing in parallel with no concern for any potential interference. To discipline those invocations, assume a synchronisation behaviour modelled by the control process $C \stackrel{\text { def }}{=} m_{r}(x) \cdot \bar{n}_{r}\langle x\rangle+$ $m_{w}(x) \cdot \bar{n}_{w}\langle x\rangle$, where the choice operator in fact represents a mutual exclusion lock on those methods. Then the parallel composition of the two processes, $(\nu n)(C \mid F)$, will be weakly bisimilar to $R \stackrel{\text { der }}{=} m_{r}(x) \cdot M_{r}\langle x\rangle+m_{w}(x) \cdot M_{w}\langle x\rangle$, as expected. More complicated and generic method exclusion relations, besides mutual exclusion locks, can be simply modelled and composed in exactly the same way, once the $\kappa$-calculus ( [Zhang02a]) is used. The $\kappa$-calculus is an extended calculus which welds the mobility power of the $\pi$-calculus with the synchronisation expressiveness of the algebra of exclusion ([Noble00]). In this paper the responsive bisimulation is presented in the polar $\pi$-calculus, an asynchronous $\pi$-calculus with polars, which is a simplified version of the $\kappa$-calculus and allows us to focus on those features essential for responsive bisimulation, without the full complexity of the $\kappa$-calculus. The following "real world" scenario illustrates the need for responsive bisimulation: 
In the mailroom of a commercial office building, the property manager uses internal mail to send bills to her tenants and collect payments. Each tenant has a locked mailbox, located either on the mailroom wall and accessible from outside of the mailroom by the tenant, or on the door of the tenant's suite to which a postman delivers mail from the mailroom. Suppose the manager only wants to know about the arrival of payments, and classify tenants who pay the bill on time as "good" tenants. She does not care whether the mailboxes are in the mailroom or on their doors. However, existing bisimulations distinguish between cases where the mailroom mailboxes are cleared or not, as the manager can observe this; however she cannot distinguish between mail being cleared or not from the door-based mailboxes. We aim to provide a model which is able to treat the mailroom and door-based mailboxes as equivalent.

Let us model this scenario. The process $\mathrm{O}_{1}$ and $\mathrm{O}_{2}$ illustrated in Figure 1 are composite processes representing two different versions of a mailbox locked with a key $\kappa$. The only difference between them is that $O_{1}$ has an extra "empty" control $\mathrm{Ctrl}_{e}$ (the postman) which does nothing but forward whatever message received from channel $m$ to the next control $\mathrm{Ctrl}_{l}$ (the locked mailbox). The body (a tenant) of these two can always give the same response (a payment) if fed with the same message (a bill). If an unlocking signal is received via channel $\kappa$, both $O_{1}$ and $O_{2}$ can accept incoming messages and process them immediately. If some message arrives before the unlocking, $O_{1}$ will store it in an internal buffer (the door mailbox), but $O_{2}$ will leave the message in the external buffer (the mailroom) as it was. In both cases, the processing will only continue after unlocking.



(a)

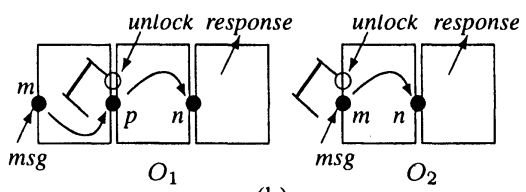

(b)

Figure 1. Two objects with the same responsive behavour

For a client (the manager) who is sending the message, the behaviour of the target object can be measured only by observing how it responds. Since the responses $O_{1}$ and $\mathrm{O}_{2}$ can give are the same, their behaviour are identical in the client's eyes. However, this behavioural similarity cannot be captured by most of the known behavioural equivalences, since in some stage $O_{1}$ can perform an input action from the channel $m$ while $\mathrm{O}_{2}$ cannot. Even the weak barbed-equivalence, one of the weakest, is too strong for them, since $O_{1} \mid R$ and $O_{2} \mid R$ are not weakly barbed-bisimilar for some $R$, such as $R \stackrel{\text { def }}{=} \bar{m}\langle\tilde{v}\rangle$. The responsive bisimulation recovers this equivalence.

\section{The polar $\pi$-calculus ( $\pi_{p}$-calculus)}

As in the asynchronous $\pi$-calculus ([Amadio96, Hütte196]), output in the polar $\pi$-calculus ( $\pi_{p}$-calculus) is non-blocking, and is not used as a prefix or choice point. Similar to [Odersky95], each name $m$, which can be considered as a reference to a 
communication channel, has an input polar $m$ and an output polar $m$, which in turn can be considered as the input port from, and the output port to, the channel $m$. The main difference from [Amadio96] and [Odersky95] is that in the $\pi_{p}$-calculus, only output polars can be transmitted through a communication channel. [Ravara97, Merro00] and others have adopted a similar restriction, but in the $\pi_{p}$-calculus this restriction is enforced syntactically. As a consequence, only output polar substitution can be caused by input prefix, while in [Odersky95] substitution may involve names with both polarities and in [Amadio96] it may affect both input and output usage of a name.

One of the advantages in using polars to enforce this restriction rather than using the implicit restriction as in [Ravara97] and [Merro00], is the simplification afforded in describing and proving some properties of bisimulations, such as bisimilarity between process $P$ and $Q$ being preserved by $m(\bar{x}) \cdot P$ and $m(\bar{x}) \cdot Q$ for all input prefixes $\hbar(\bar{x})$.

The notion of polarised ports exists in many forms of communication. For example, an email account consists of a mailbox (input polar) and an email address (output polar). You may send the address to other people for them to send you message, but you can neither send your mailbox nor tell someone "receive my next email using this address". Moreover, if input polars can be transmitted, then the behaviour of a process will no longer be predictable from its structure. For object modelling, the prohibition on transmitting input polars respects the idea that object identity and method ownership cannot be transferred from one object to another (see Section 5).

The Syntax. Let $\mathcal{N}$ be the set of all names, ranged over by name expressions $m, n, u, v$ and variables $x, y$. Let ${ }^{+} \mathcal{N} \stackrel{\text { def }}{=}\{n: n \in \mathcal{N}\}$ and $\mathcal{N} \stackrel{\text { def }}{=}\{n: n \in \mathcal{N}\}$ be the sets of input polars and output polars respectively. Let polar expressions $a, b$ and variable $w$ range over the set of all polars, ${ }^{+} \mathcal{N} \cup \mathcal{N}$. $\tilde{r}$ is the abbreviation for $r_{1}, r_{2}, \cdots, r_{n}$. The generic process terms $P$ in the $\pi_{p}$-calculus are generated by the following grammar:

$$
\begin{gathered}
P::=m(\tilde{\tilde{u}})|(\nu \tilde{n}) P| P_{1}\left|P_{2}\right| ! B \mid G \\
G::=0|B|(\nu \tilde{n}) G \mid G_{1}+G_{2}, \quad B::=m(\tilde{\bar{x}}) . P
\end{gathered}
$$

The set of all actions a process may take is specified by

$$
\alpha::=m(\tilde{u})|(\nu \tilde{v}) m(\tilde{u}\rangle| \tau, \quad \text { where } \quad \tilde{v} \subseteq \tilde{u} \text { and } m \notin \tilde{v} .
$$

Here 0 is the inactive (terminated) process; $m\langle\tilde{u}\rangle$ is the output action which sends output polars $\tilde{u}$ into the channel $m ;(\nu \tilde{n}) P$ binds the set of names $\tilde{n}$, and therefore both polars of each of those names, within the scope of $P ; P_{1} \mid P_{2}$ indicates two processes run in parallel; $B$ is an input-guarded process; $! B$ is replication and $G$ is exclusive choice, both constructed from input-guarded processes.

As usual, auxiliary functions $f n, b n$ and $n$ are used to identify the sets of free, bound and all names, respectively, of a term or action. Specific functions to identify polars are also needed. For example, the function fin identifies those names whose input polar freely occurs in a term or action.

A formalised mapping between the $\pi_{p}$-calculus and the asynchronous $\pi$-calculus of [Amadio96], and that between the $\pi_{p}$-calculus and the polarised $\pi$-calculus of [Odersky95], can be found in [Zhang02b]. 
Table 1. Structural congruence rules for the polar $\pi$-calculus

\begin{tabular}{clll}
\hline Summation & & \\
str-SUM1: & $P_{1}\left|0 \equiv P_{1} ; \quad P_{1}\right| P_{2} \equiv P_{2} \mid P_{1} ;$ & $P_{1}\left|\left(P_{2} \mid P_{3}\right) \equiv\left(P_{1} \mid P_{2}\right)\right| P_{3}$ \\
str-SUM2: & $G_{1}+0 \equiv G_{1} ; \quad G_{1}+G_{2} \equiv G_{2}+G_{1} ;$ & $G_{1}+\left(G_{2}+G_{3}\right) \equiv\left(G_{1}+G_{2}\right)+G_{3}$ \\
Scope & & \\
str-SCP1: & $(\nu \tilde{n}) P \equiv P$, if $\tilde{n} \cap f n(P)=\emptyset ;$ & $(\nu \tilde{n}) G \equiv G$, if $\tilde{n} \cap f n(G)=\emptyset$ \\
str-SCP2: & $(\nu m) m(\tilde{y}) \equiv 0 ;$ & $(\nu m) \tilde{m}(\tilde{\tilde{x}}) . P \equiv 0$ \\
str-SCP3: & $(\nu \tilde{m})(\nu \tilde{n}) P \equiv(\nu \tilde{m}, \tilde{n}) P ;$ & $(\nu \tilde{m})(\nu \tilde{n}) P \equiv(\nu \tilde{n})(\nu \tilde{m}) P$ \\
str-SCP4: & $(\nu \tilde{n}) P_{1} \mid P_{2} \equiv(\nu \tilde{n})\left(P_{1} \mid P_{2}\right)$, & if $\tilde{n} \cap f n\left(P_{2}\right)=\emptyset$ \\
& $(\nu \tilde{n}) G_{1}+G_{2} \equiv(\nu \tilde{n})\left(G_{1}+G_{2}\right)$, & if $\tilde{n} \cap f n\left(G_{2}\right)=\emptyset$ \\
str-REN: & $(\nu \tilde{n}) P \equiv(\nu \tilde{m})(P\{\tilde{m} / \tilde{n}\})$, & if $\tilde{m} \cap f n(P)=\emptyset$ \\
\hline
\end{tabular}

Table 2. Labelled transition rules for process terms in the polar $\pi$-calculus

\begin{tabular}{|c|c|c|c|c|}
\hline \multirow{2}{*}{ tr-OUT: } & & $\stackrel{P(\tilde{u})}{\longrightarrow} P^{\prime}, \quad m \notin \tilde{v}$ & \multirow{2}{*}{ tr-IN: } & \\
\hline & $m\langle\tilde{u}\rangle \stackrel{m(\tilde{u})}{\longrightarrow} 0_{P}{ }^{\prime}$ & $(\nu \tilde{v}) P \stackrel{(\nu \tilde{v}) m\langle\tilde{u}\rangle}{\longrightarrow} P^{\prime}$ & & $\overline{m(\tilde{\bar{x}}) \cdot P \stackrel{m(\tilde{u})}{\longrightarrow} P\{\tilde{\tilde{u}} / \tilde{\bar{x}}\}}$ \\
\hline \multirow{2}{*}{ tr-RES: } & \multicolumn{2}{|c|}{$\underline{P} \stackrel{\alpha}{\rightarrow} P^{\prime}, \quad \tilde{n} \cap f n(\alpha)=\emptyset$} & \multirow{2}{*}{ tr-REP: } & $B \stackrel{m(\tilde{u})}{\longrightarrow} P$ \\
\hline & $(\nu \tilde{n}) P \stackrel{\alpha}{\rightarrow}($ & $\tilde{n}) P^{\prime}$ & & $! B \stackrel{m(\tilde{u})}{\longrightarrow} P \mid ! B$ \\
\hline \multirow{2}{*}{ tr-PARL: } & $P \stackrel{\alpha}{\rightarrow} P^{\prime}$ & & \multirow{2}{*}{ tr-CHOI: } & $G_{1} \stackrel{m(\tilde{u})}{\longrightarrow} P$ \\
\hline & $P\left|Q \stackrel{\alpha}{\rightarrow} P^{\prime}\right| Q$ & & & $G_{1}+G_{2} \stackrel{m(\tilde{u})}{\longrightarrow} P$ \\
\hline tr-INTL: & $\frac{P \stackrel{(\nu \tilde{v}) m\langle\hat{u}\rangle}{\rightarrow} P^{\prime}}{(\nu m) P \stackrel{\tau}{\rightarrow}(\nu}$ & $\frac{P^{\prime} \stackrel{m(\tilde{u})}{\longrightarrow} P^{\prime \prime}}{m)(\nu \tilde{v}) P^{\prime \prime}}$ & tr-STRUC: & $\frac{P_{1}^{\prime} \equiv P_{1}, \quad P_{1} \stackrel{\alpha}{\rightarrow} P_{2}, \quad P_{2} \equiv P_{2}^{\prime}}{P_{1}^{\prime} \stackrel{\alpha}{\rightarrow} P_{2}^{\prime}}$ \\
\hline
\end{tabular}

The Semantics. The structural equivalences and labelled transitions are shown in Table 1 and Table 2. They are standard except the rule tr-INTL, which gives the meaning of an internal action $\tau$. A variant of this rule can be written as:

$$
\frac{P \stackrel{(\nu \tilde{v}) m(\tilde{u}\rangle}{\longrightarrow} P^{\prime}, Q \stackrel{m(\tilde{u})}{\longrightarrow} Q^{\prime}, \quad \tilde{v} \cap f n(Q)=\emptyset}{(\nu m)(P \mid Q) \stackrel{\tau}{\rightarrow}(\nu m)(\nu \tilde{v})\left(P^{\prime} \mid Q^{\prime}\right)} .
$$

The name restriction $(\nu m)$ is necessary for preserving any bisimulation involving $\tau$ action, under the input prefixing $m(\tilde{\tilde{x}}) . P$ in which substitution can only occur for the output polar of the name $m$, rather than both polars.

Definition 1 Weak transitions are defined as:

$P \stackrel{\tau}{\Rightarrow} P^{\prime}$ iff $P(\stackrel{\tau}{\rightarrow})^{*} P^{\prime}$, where * indicates zero or finitely many $\tau$-transitions;

$P \stackrel{\alpha}{\Rightarrow} P^{\prime}$ iff $P \stackrel{\tau}{\Rightarrow} \stackrel{\alpha}{\rightarrow} \stackrel{\tau}{\Rightarrow} P^{\prime}$, where $\alpha \neq \tau$.

The reduction relation, a familiar concept in this literature, is defined in a nonstandard way in the $\pi_{p}$-calculus: 
Definition 2 The strong and weak reduction relations are defined respectively as: $P \rightarrow P^{\prime} \quad$ iff $(\nu m) P \stackrel{\tau}{\rightarrow}(\nu m) P^{\prime}$, for some $m$; $P \Rightarrow P^{\prime} \quad$ iff $(\nu m) P \stackrel{\tau}{\Rightarrow}(\nu m) P^{\prime}$, for some $m$.

Clearly, $P \stackrel{\tau}{\rightarrow} P^{\prime}$ implies $P \rightarrow P^{\prime}$, and $P \stackrel{\tau}{\Rightarrow} P^{\prime}$ implies $P \Rightarrow P^{\prime}$, and therefore, another variant of the rule tr-INTL can be written as:

$$
\frac{P \stackrel{(\nu \tilde{v}) m\langle\tilde{u}\rangle}{\longrightarrow} P^{\prime}, Q \stackrel{m(\tilde{u})}{\longrightarrow} Q^{\prime}, \tilde{v} \cap n(Q)=\emptyset}{P \mid Q \rightarrow(\nu \tilde{v})\left(P^{\prime} \mid Q^{\prime}\right)},
$$

which looks more like the usual communication rule for the $\pi$-calculus. Making the distinction between internal action and reduction is also necessary for the new bisimulation relation, as we will find out later.

Definition 3 The strong commitments of process $P$ are defined as:

$P$ can commit the action $\alpha$, denoted as $P \downarrow \alpha$, if $P \stackrel{\alpha}{\rightarrow} P^{\prime}$ for some $P^{\prime}$;

$P$ can commit on input polar $t m$, denoted as $P \downarrow m$, if $P \downarrow m(\tilde{u})$;

$P$ can commit on output polar $m$, denoted as $P \downarrow m$, if $P \downarrow(\nu \tilde{v}) m\langle\tilde{u}\rangle$;

The weak commitments $\Downarrow$, are obtained by replacing $\rightarrow$ with $\Rightarrow$ and $\downarrow$ with $\Downarrow$ throughout.

Definition 4 Process $P^{\prime}$ is a derivative of $P$, if there exists some finite sequence of actions $\alpha_{1}, \alpha_{2}, \cdots, \alpha_{n}$, such that $P \stackrel{\alpha_{1}}{\rightarrow} \stackrel{\alpha_{2}}{\rightarrow} \ldots \stackrel{\alpha_{n}}{\rightarrow} P^{\prime}$.

\section{Responsive bisimulation in the polar $\pi$-calculus}

As pointed out at the end of Section 2, the barbed equivalence, even in its weak form, is too strong for compositional objects. We first review the version of [Amadio96] for an asynchronous $\pi$-calculus before leading on to responsive bisimulation. It is derived from the barbed bisimulation ([MilSan92, Sangi92]), a rather weak relation which traces the state changes of a process during the course of reductions and observes which channels are available for communication.

Definition 5 (Barbed bisimulation) A symmetric relation $\mathcal{S}$ on $P$-terms is a (strong) barbed bisimulation if whenever $P \mathcal{S} Q$ then $P \downarrow$ a implies $Q \downarrow a$ for $a \in \mathcal{N}$, and $P \rightarrow P^{\prime}$ implies $\exists Q^{\prime}$ such that $Q \rightarrow Q^{\prime}$ and $P^{\prime} \mathcal{S} Q^{\prime}$.

Let $\sim_{b}$ be the largest strong barbed bisimulation. The notion of weak barbed bisimulation $\approx_{b}$ is obtained by replacing the transition $\downarrow$ with $\Downarrow$, and $\rightarrow$ with $\Rightarrow$ throughout.

Since barbed bisimulation cannot identify what messages are communicated, it is too rough to measure processes' behaviour.

Definition 6 (Barbed equivalence) Let $R$ be an arbitrary process, the strong and weak barbed equivalence are defined respectively as: $P \simeq_{b} Q$ if $\forall R .\left(R\left|P \sim_{b} R\right| Q\right)$;

$$
P \approx_{b} Q \text { if } \forall R .\left(R\left|P \approx_{b} R\right| P\right) \text {. }
$$

For our purposes, the weak barbed equivalence fails in at least three ways: (a) it cannot distinguish between a message sent out from the target process and a message 
Table 3. Rules for localised messaging

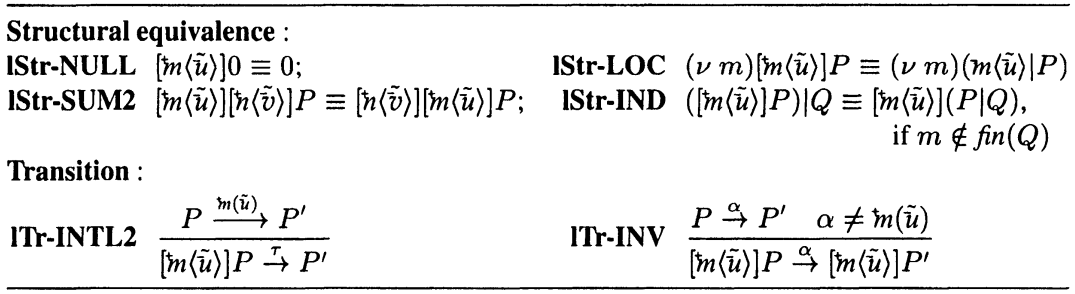

sent by another agent but buffered in the environment; (b) it cannot prevent input name clashes between the testing environment and the processes being tested; (c) it treats synchronisation actions occurring in public channels as single step reduction, and therefore cannot match them with incomplete synchronisations which have input delays. A different observation technique is needed. We begin with the $o \tau$-bisimulation similar to that in [Amadio96]:

Definition 7 (o $\tau$-bisimulation) The o $\tau$-bisimulation is a symmetric relation $\mathcal{S}$ on processes, for which whenever $P \mathcal{S} Q$ then $P \stackrel{\alpha}{\rightarrow} P^{\prime}$ implies $Q \stackrel{\alpha}{\rightarrow} Q^{\prime}$ and $P^{\prime} \mathcal{S} Q^{\prime}$, where $\alpha$ is a non-input action and $b n(\alpha) \cap f n(Q)=\emptyset$.

The weak or-bisimulation is obtained by replacing $\stackrel{\alpha}{\rightarrow}$ with $\stackrel{\alpha}{\Rightarrow}$ throughout. We denote the largest (strong) o $\tau$-bisimulation as $\sim_{o \tau}$, and the largest weak o $\tau$-bisimulation as $\approx_{o \tau}$.

The $o \tau$-bisimulation gives a measurement on processes' states by observing available reductions and output actions, but cannot determine how a process responds to incoming messages, since input actions are not observed. To determine responsive behaviours, we introduce a new term for specifying input messages.

Notation 8 We extend the process syntax with an auxiliary $P$-term $[m\langle\tilde{u}\rangle] P$, the localisation of the sent message $m\langle\tilde{u}\rangle$ with process $P$. Properties for this term are shown in Table 3.

The term $[m\langle\tilde{u}\rangle] P$ is not for modelling processes, but only designed to express responsive bisimulation relations between processes. It couples process $P$ with the message $\tilde{u}$ which is buffered in channel $m$ and unobservable from outside, so that it cannot be mistaken by external observers as an output from $P$. However the output polar $m$ is not hidden by this term. This issue will be discussed further in Section 6 .

The rule ITr-INTL2 adds a new case for introducing a $\tau$ action. Unlike rule tr-INTL, the name restriction is not required here. However, since only the input polar, $t m$, of the channel name $m$ is involved, the preservation of $\tau$ actions is maintained by input prefixing.

Definition 9 (Responsive equivalence) Let $\mathcal{T}[$.] be the responsive testing context with syntax $\mathcal{T}::=[] \mid.[m\langle\tilde{u}\rangle] \mathcal{T}$. We define strong and weak responsive equivalences as:

$$
P \simeq_{r} Q \quad \text { iff } \forall \mathcal{T} .\left(\mathcal{T}[P] \sim_{o \tau} \mathcal{T}[Q]\right) ; \quad P \approx_{r} Q \text { iff } \forall \mathcal{T} .\left(\mathcal{T}[P] \approx_{o \tau} \mathcal{T}[Q]\right) .
$$


This definition is clear about the meaning of equivalence in responsive behaviour, but is not so useful for proving equivalence, since it requires the exhaustive testing over the infinite set of responsive testing contexts. A more practical definition is the r1-bisimulation.

Definition 10 A strong (or weak) rl-bisimulation is a strong (or weak, respectively) o $\tau$-bisimulation $\mathcal{S}$ such that whenever $P \mathcal{S} Q$ then $[m\langle\tilde{u}\rangle] P \mathcal{S}[m\langle\tilde{u}\rangle] Q$ for all $[m\langle\tilde{u}\rangle]$.

We denote the largest strong rl-bisimulation as $\sim_{r 1}$, and the largest weak $r l$ bisimulation as $\approx_{r 1}$.

It is easy to verify that $O_{1} \approx_{r 1} O_{2}$ holds for the example of Figure 1 . The r1-bisimulation provides a test platform and measures behavioural equivalence from outside of target processes.

While responsive equivalence and r1-bisimulation provide a good base for describing similarities of responsive behaviours, it can tell little about why or when two processes may offer similar behaviours. For closer study, we need an inside view observing input actions.

Definition 11 (Responsive bisimulation) $A$ (strong) responsive bisimulation is a (strong) or-bisimulation $\mathcal{S}$ such that whenever $P \mathcal{S} Q$ then $P \stackrel{m(\tilde{u})}{\longrightarrow} P^{\prime}$ implies either $Q \stackrel{m(\tilde{u})}{\longrightarrow} Q^{\prime}$ and $P^{\prime} \mathcal{S} Q^{\prime}$, or $Q \stackrel{\tau}{\rightarrow} Q^{\prime}$ and $P^{\prime} \mathcal{S}[m(\tilde{\tilde{u}})] Q^{\prime}$.

The weak version is obtained by replacing transitions with weak transitions everywhere. We denote the largest strong and weak responsive bisimulation by $\sim_{r}$ and $\approx_{r}$ respectively. Clearly, $\sim_{r} \subseteq \approx_{r}$.

For the example of Figure 1 , we can easily verify that $O_{1} \approx_{r} O_{2}$. In general, the two views mentioned in the introduction are equivalent:

Lemma 12 responsive bisimulation, rl-bisimulation and responsive equivalence all coincide: $\sim_{r} \equiv \sim_{r 1} \equiv \simeq_{r}$ and $\approx_{r} \equiv \approx_{r 1} \equiv \approx_{r}$.

\section{Properties of responsive bisimulation}

We now investigate some formal properties of responsive bisimulation.

Lemma 13 Responsive bisimulations are equivalences. That is, they are reflexive, symmetric and transitive.

Proposition 14 Responsive bisimulation is preserved by output polar substitution, input prefix, restriction, localisation and choice. That is, let $\mathcal{S}$ be either $\sim_{r}$ or $\approx_{r}$, then, $P S Q$ implies

1. $P \sigma \mathcal{S} Q \sigma$ for all $\sigma=\{\tilde{u} / \tilde{x}\}$

2. $m(\tilde{\tilde{u}}) \cdot P \operatorname{S} m(\tilde{\tilde{u}}) \cdot Q$ for all $m(\tilde{\tilde{u}})$;

3. $\quad(\nu \tilde{v}) P \mathcal{S}(\nu \tilde{v}) Q$ for all $\tilde{v}$;

4. $[m\langle\tilde{u}\rangle] P \mathcal{S}[m\langle\tilde{u}\rangle] Q$ for all $[m\langle\tilde{u}\rangle]$.

and, $G_{1} \mathcal{S} G_{2}$ implies $\left(G_{1}+G\right) \mathcal{S}\left(G_{2}+G\right)$ for all $G$.

A problem is apparent: the responsive bisimulation is not preserved by parallel composition in general. For example, in the Figure 1 we have $O_{1} \approx_{r} O_{2}$, but 
$\left(O_{1} \mid O_{3}\right) \not z_{r}\left(O_{2} \mid O_{3}\right)$ for $O_{3} \stackrel{\text { def }}{=} m . R$, because the occurrence of input polar $m$ in $O_{3}$ has changed the ability of $O_{1}$ to receive messages on $m$. However, the purpose of our study is object modelling, where the ownership of each input port should be unique. For example, the identity of an object is unique and each method of each object is also uniquely identified so that messages are delivered to their correct destination. In general, each input polar has a restricted scope (or ownership), and is never exported outside this scope. When responsive bisimulation is restricted to the object modelling domain, its preservation in parallel composition can be guaranteed. To show this, we formalise the restriction on input polars.

Definition 15 (Safe process) A process $P$ is safe for Env, and the environment Env is said to be safe for $P$, if $\operatorname{fin}(P) \cap \operatorname{fin}(E n v)=\emptyset$.

We may call $P$ a safe process, when the behaviour of $P$ is only considered within environments safe for $P$.

A process $P$ is autonomous if $\operatorname{fin}(P)=\emptyset$.

Lemma 16 Evolution preserves process safety. That is, all derivatives of a process $P$ safe for environment Env are safe for Env and its derivatives. An autonomous process and all its derivatives are safe in any system.

When modelling objects, all method bodies can be considered as autonomous; even an object itself is initially autonomous until creation. After creation, input can only be performed via channels that were initially private.

Proposition 17 Responsive bisimulation is preserved by parallel composition for safe processes: let $\mathcal{S}$ be either $\sim_{r}$ or $\approx_{r}$, then $P_{1} \mathcal{S} P_{2}$ implies $\left(P_{1} \mid P\right) \mathcal{S}\left(P_{2} \mid P\right)$ for all $P$ satisfying $\left(\operatorname{fin}\left(P_{1}\right) \cup \operatorname{fin}\left(P_{2}\right)\right) \cap \operatorname{fin}(P)=\emptyset$.

Proposition 18 Replication preserves responsive bisimulations for autonomous processes. That is, let $\mathcal{S}$ be either $\sim_{r}$ or $\approx_{r}, P_{1}$ and $P_{2}$ be autonomous processes, then $P_{1} \mathcal{S} P_{2}$ implies $\left(! n(\tilde{\tilde{x}}) \cdot P_{1}\right) \mathcal{S}\left(! n(\tilde{\tilde{x}}) . P_{2}\right)$ for all input prefix $\hbar(\tilde{\tilde{x}})$.

Corollary 19 For autonomous processes, responsive bisimulations are congruences.

An interesting result which demonstrates responsive equivalence of an object and a wrapped version of it, is :

Proposition $20 P \approx_{r}(\nu n)(\operatorname{tm}(\tilde{\tilde{x}}) \cdot n\langle\tilde{x}\rangle \mid P\{\hbar / \hbar m\})$ for all $P$ and $m$, where $n \notin f n(P)$.

\section{Discussion}

Privatise message versus privatise port. It is worth emphasising that the $[m\langle\tilde{u}\rangle]$ in the term $[m\langle\tilde{u}\rangle] P$ hides neither polar $m$ nor $m$, but the message $\tilde{u}$ which the input polar $m$ is yet to consume. The output polar $m$ remains public, and any message emitted via $m$ by $P$ itself must be considered as part of the observable behaviour of $[m\langle\tilde{u}\rangle] P$. There is a difference between $[m\langle\tilde{u}\rangle] P$ and $m\langle\tilde{u}\rangle \mid P$ : in the former, $[m\langle\tilde{u}\rangle]$ is a buffered message arriving from the channel $m$, waiting for $P$ or its derivatives to 
pick it up (though they may not); in the latter, the $m\langle\tilde{u}\rangle$ is an outgoing message to be buffered in the channel $m$, and therefore is not distinguishable from an output of $P$.

The input polar $m$ appearing in $[m\langle\tilde{u}\rangle]$ will not be affected by an output polar substitution, just as one cannot change the destination address of mail after it is sent. Further intuition for the meaning of the term $[m\langle\tilde{u}\rangle] P$ may be perceived from the immediate deduction from Proposition 20: $[m\langle\tilde{u}\rangle] P \approx_{r}(\nu n)(\operatorname{lm}(\tilde{\tilde{x}}) \cdot n\langle\tilde{\tilde{x}}\rangle|n\langle\tilde{u}\rangle| P\{\hbar / m\})$.

Delay of input versus delay of output. The asynchronous bisimulation $\sim_{a}$ (or $\approx_{a}$ for the weak version), is defined in [Amadio96] as an $o \tau$-bisimulation $\mathcal{S}$ for which whenever $P \mathcal{S} Q$ then $P \stackrel{m(\tilde{u})}{\longrightarrow} P^{\prime}$ implies either $Q \stackrel{m(\tilde{u})}{\longrightarrow} Q^{\prime}$ and $P^{\prime} \mathcal{S} Q^{\prime}$, or $Q \stackrel{\tau}{\rightarrow} Q^{\prime}$ and $P^{\prime} \mathcal{S}\left(m\langle\tilde{u}\rangle \mid Q^{\prime}\right)$. It emphasises the possible delay of message output (or more precisely, delays during delivery), and considers message retransmission with the same communication channel as ignorable. In the view of $\mathrm{OO}$ systems, the delay in delivery is neither visible nor controllable for either sender or receiver. The responsive bisimulation, on the other hand, is quite natural for compositional objects since it concentrates on the delay of input, which is controllable by the receiver, and as pointed out by [McHale94] and [Zhang98b], can provide a synchronisation control point for the behavioural composition of objects. The $\mathrm{Ctrl}_{e}$ and $\mathrm{Ctrl}_{l}$ in Figure 1 are examples of these kind of controls.

Asynchronous and responsive bisimulations overlap, but neither contains the other. For example, the processes $m . m \mid P$ and $P$ are weakly asynchronous bisimilar when $m \notin f i n(P)$, but not weakly responsive bisimilar; while the processes $(\nu n)(m . n \mid k . n . P)$ and $k . m . P$ are weakly responsive but not asynchronous bisimilar. Details and proofs about the relation between responsive bisimulation and other well known bisimulations can be found in [Zhang02b].

\section{Conclusion}

This paper has presented the responsive bisimulation, which captures an equivalence between compositional concurrent objects, based on their response to inputs which may be delayed. For object systems, where input name clash can be eliminated, the responsive bisimulation is a congruence.

The responsive bisimulation can be understood in different ways. Apart from the view of "input delay", another view is that, when testing the behaviour of the target object, or black box, the only precondition we need to know is what messages have been provided to it, and the only postcondition we should examine is the response from the target object. We have proven these different views are equivalent.

With the responsive bisimulation, we can have a broader and more generic study of the behaviour of concurrent components, where existing bisimulations fail to give us the desired equivalence. Our approach enables us to establish a theory of concurrent objects with elegant compositional properties and provides a semantic basis for an extension to concurrent object-oriented programming languages. 


\section{References}

[Aksit92] Mehmet Aksit and Lodewijk Bergmans. (1992). "Obstacles in Object-oriented Software Development", Proc. of OOPSLA'92, ACM SIGPLAN Notices vol. 27, pages 341-358, New York.

[Amadio96] Roberto M. Amadio, Ilaria Castellani and Davide Sangiorgi. (1996). "On Bisimulations for the Asynchronous $\pi$-calculus", in Proc. of CONCUR'96, LNCS vol. 1119, Springer Verlag.

[Holmes97] David Holmes, James Noble, John Potter. (1997). "Aspects of Synchronisation", in Christine Mingins, Roger Duke and Bertrand Meyer, editors, Proc. of TOOLS 25 (TOOLS Pacific'97), pages 7-18, Melbourne, Australia.

[Hüttel96] Hans Hüttel and Josva Kleist. (1996). "Objects as mobile processes", Aalborg University.

[Jones93] Cliff B. Jones. (1993). "A $\pi$-calculus Semantics for an Object-based Design Notation", in E. Best, editor, Proc. of CONCUR'93, LNCS vol. 715, pages 158172. Springer Verlag.

[McHale94] Ciaran McHale. (1994). "Synchronisation in Concurrent, Object-oriented Languages: Expressive Power, Genericity and Inheritance", $\mathrm{PhD}$. Thesis, Department of Computer Science, Trinity college, University of Dublin, Ireland.

[Merro00] Massimo Merro, Josva Kleist and Uwe Nestmann. (2000). "Local $\pi$-Calculus at Work: Mobile Object as Mobile Processes", In Proc. of IFIP TCS2000, Sendai, Japan. LNCS vol. 1872, pages 390-408, Springe.

[Milner92] R. Milner, J. Parrow, D. Walker. (1992). "A Calculus of Mobile Process" (Parts I and II), Journal of Information and Computation, 100:1-77.

[MilSan92] Robin Milner and Davide Sangiorgi. (1992). "Barbed Bisimulation", in W. Kuich, editor, Proceeding of 19th ICALP, LNCS vol. 623, Springer Verlag.

[Milner99] Robin Milner. (1999). "Communicating and Mobile Systems: the $\pi$-calculus", Cambridge University Press.

[Noble00] James Noble and John Potter. (2000). "Exclusion for Composite Objects", In Proc. of OOPSLA 2000, Minneapolis, Minnesota USA, ACM press.

[Odersky95] Martin Odersky. (1995). "Polarized Name Passing", in Proc. of FST\& TCS'95, Bangalore, India.

[Ravara97] António Ravara and Vasco T. Vasconcelos. (1997). Behavioural types for a calculus of concurrent objects. In C. Lengauer, M. Griebl, and S. Gorlatch, editors, Proc. of Euro-Par'97, LNCS 1300, pages 554-561. Springer-Verlag.

[Sangi92] Davide Sangiorgi. (1992). "Expressing Mobility in Process Algebras: First-Order and Higher-Order paradigms", $\mathrm{PhD}$ thesis, Computer Science Department, University of Edinburgh, UK.

[Sangi96] Davide Sangiorgi. (1996). "An Interpretation of Typed Objects into Typed $\pi$ calculus", INRIA Technical Report RR-3000.

[Schne97] Jean-guy Schneider and Markus Lumpe. (1997). "Synchronizing Concurrent Objects in the Pi-Calculus", Proceedings of Langages et Modèles à Objets '97, Roland Ducournau and Serge Garlatti (Ed.), pp.61-76, Hermes, Roscoff.

[Walker95] David Walker. (1995). "Objects in the $\pi$-Calculus", Information and Computation, 116(2):253-271. 
[Zhang97] Xiaogang Zhang and John Potter. (1998). "Class-based models in $\pi$-calculus", in Christine Mingins, Roger Duke and Bertrand Meyer, editors, Proc. of TOOLS 25 (TOOLS Pacific'97), Melbourne, Australia, 24th-27th November 1997, pages 238-251, IEEE Computing Society Press.

[Zhang98a] Xiaogang Zhang and John Potter. (1998). "Compositional Concurrency Constraints for Object Models in $\pi$-calculus", Technical Report C/TR-9804, Macquarie University, Sydney, Australia.

[Zhang98b] Xiaogang Zhang and John Potter. (1998). "A Composition Approach to Concurrent Objects", in Jian Chen, Mingshu Li, Christine Mingins and Bertrand Meyer, editors, Proc. of TOOLS 27 (TOOLS Asia'98), Beijing, China, 22nd-25th September 1998, pages 116-126, IEEE Computing Society Press.

[Zhang02a] Xiaogang Zhang and John Potter. (20012). "A Constraint Description Calculus for Compositional Concurrent Objects", Technical report UNSW-CSE-TR-0204.

[Zhang02b] Xiaogang Zhang and John Potter. (2002). "The Responsive Bisimulations in the polar $\pi$-calculus", Technical report UNSW-CSE-TR-0203. 